\title{
MODELAGEM MATEMÁTICA: DOS ENTENDIMENTOS ÀS FINALIDADES
}

\author{
BIANCA DE OLIVEIRA MARTINS ${ }^{1}$ \\ LOURDES MARIA WERLE DE ALMEIDA²
}

MATHEMATICAL MODELING: FROM UNDERSTANDINGS TO PURPOSE

\section{RESUMO}

Neste artigo, temos por objetivo investigar a interrogação: o que se entende por modelagem matemática no contexto dos trabalhos publicados nos livros da International Conference on Teaching Mathematical Modelling and Applications (ICTMA)? Tendo por norte os pressupostos da abordagem fenomenológica exploramos as nuances do modo como 0 fenômeno se revela nos anais da ICTMA relativos ao período de 2010 a 2017. Dados foram coletados em cinquenta e sete trabalhos publicados na conferência, os quais foram selecionados a partir da aplicação subsequente dos critérios: os textos deveriam contemplar o termo modelagem no título ou no resumo do texto, os autores que publicaram em pelo menos três edições diferentes do ICTMA e, a descrição de uma atividade de modelagem matemática. Resultados são evidenciados a partir da manifestação de quatro núcleos de ideias que evidenciam o entendimento de modelagem matemática revelado nas publicações, ou seja, modelagem matemática: como forma de resolver um problema; como prática pedagógica; como possibilidade de resolver na sala de aula problemas autênticos; como possibilidade de promover pensamento crítico.

Palavras-chave: Educação Matemática. Fenomenologia. ICTMA.

\section{ABSTRACT}

In this article we present a qualitative investigation that asks: what is meant by mathematical modeling in the context of the articles published in the books of International Conference on Teaching Mathematical Modeling and Applications? The methodological itinerary for the research follows the direction of a phenomenological investigation, which aims to understand the phenomenon as it manifests. To bring to the discussion elements that also cover an international panorama, we turn our attention to the proceedings of the ICTMA on the period from 2010 to 2017. From the definition of some criteria, we selected fifty-seven articles. The analysis of these articles led to four nuclei of ideas that say that mathematical modeling can be understood in these articles: as a way to solve a problem; as a pedagogical practice; as a possibility to solve authentic problems in the classroom; as a possibility to promote critical thinking. These groups explain different approaches, which based on theories or classroom practices, reveal what has been published in relation to mathematical modeling in the area of Mathematics Education.

Keywords: Mathematical Education. Phenomenology. ICTMA.

1 Professora Mestre em Ensino de Ciências e Educação Matemática. Universidade Estadual de Londrina - Paraná. bianca.o.martins@uel.br. ORCID: https://orcid.org/0000-0001-8506-3649.

2 Professora Doutora do Programa de Pós-Graduação em Ensino de Ciências e Educação Matemática. Universidade Estadual de Londrina Paraná. lourdes@uel.br. ORCID: http://orcid.org/0000-0001-8952-1176. 


\section{INTRODUÇÃO}

No âmbito da Educação Matemática a modelagem matemática vem ganhando espaço como temática de pesquisa bem como nas discussões relativas às práticas na sala de aula, particularmente nas aulas de matemática.

No entanto, conforme já aponta 0 trabalho de Klüber (2012), as repercussões da modelagem matemática, seja no contexto das pesquisas, seja nas práticas de ensino, da Educação Infantil ao Ensino Superior, estão baseadas em uma multiplicidade de compreensões sobre modelagem matemática. Tais compreensões, muitas vezes indicam diferentes visões de professores e alunos envolvidos em atividades dessa natureza.

Neste contexto, Bicudo e Klüber (2011), particularmente, apresentam uma metacompreensão acerca da pesquisa brasileira sobre modelagem matemática e indicam que os interesses nas investigações estão associados a várias frentes: "[...] incluídas as científicas, de cunho epistemológico e ontológico, referindo-se aos modos de produção de conhecimento e de sua constituição e avançando até as sociais, que incidem na divulgação dos resultados entre os pares e no confronto de tais resultados com a realidade educacional".

Na última década algumas pesquisas têm se dedicado à investigação das finalidades das pesquisas e das práticas de modelagem matemática nos diferentes níveis de escolaridade (NUNES et al., 2020; KLÜBER, et al., 2015) e têm se mostrado diferentes interpretações relativas aos entendimentos de modelagem matemática considerando tanto aspectos epistemológicos quanto ontológicos.

Em âmbito internacional, Jablonka, Wagner e Walshaw (2013), consideram que a diversidade de interpretações e com bases teóricas distintas pode ser um indicativo da maturidade de uma área. Sriraman e Nardi (2013) argumentam que os avanços no pensamento das comunidades de modelagem matemática se constituem justamente nestas abordagens teóricas diversificadas e complementares.

No presente artigo somos ainda movidos por uma inquietação do que tem sido veiculado relativamente ao que se entende por modelagem matemática considerando um contexto de pesquisadores e professores de diferentes comunidades mundo afora. Assim, com a finalidade de ampliar 0 debate e trazer para a discussão elementos da pesquisa em modelagem matemática que abrangem também um panorama internacional, dirigimos nossa atenção aos artigos publicados nos anais das conferências da International Conference on Teaching Mathematical Modelling and Applications (ICTMA), a partir da questão: o que se entende por modelagem matemática no contexto dos trabalhos publicados nos livros do ICTMA?

0 ICTMA é um evento pioneiro no que se refere às discussões e deliberações relativas à modelagem na Educação Matemática e sua primeira edição foi realizada no ano de 1983 na cidade Exeter na Inglaterra.

Os anais do ICTMA reúnem publicações a respeito de modelagem matemática e aplicações da matemática no ensino de matemática e suas repercussões na área de Educação Matemática incluindo pesquisas realizadas em uma diversidade de países. 0 Brasil foi sede do evento no ano de 2013. A participação de brasileiros na conferência bem como a publicação de capítulos nos livros que reúnem trabalhos apresentados e discutidos no evento tem sido frequente nas últimas décadas. Almeida e Silva (2015); Araújo e Campos (2015); Barbosa (2010); Biembengut e Hein (2010); Borba (2011); D'ambrosio (2015); Luna, Souza e Lima (2015); Oliveira e Barbosa (2013); Orey e Rosa (2015); Palharini e Almeida (2015); Palharini, Tortola e Almeida (2017); Rosa e Orey (2017) são pesquisas de autores brasileiros publicadas nos livros do ICTMA na última década. 
No presente artigo o itinerário metodológico para a pesquisa segue encaminhamentos de uma investigação fenomenológica. Portanto, a pesquisa se propõe a, de maneira direta e sem referência explícita a uma base teórica, revelar o que se entende por modelagem matemática no contexto das pesquisas publicadas nos livros dos ICTMA.

\section{DESDOBRAMENTOS METODOLÓGICOS A PARTIR DE UMA POSTURA FENOMENOLÓGICA}

Nessa pesquisa, temos como objetivo a investigação do que se entende por modelagem matemática no contexto dos trabalhos publicados nos livros da International Conference on Teaching Mathematical Modelling and Applications (ICTMA). A investigação do fenômeno associado ao entendimento de modelagem matemática nessas publicações foi feita mediante uma abordagem fenomenológica por meio dos procedimentos associados à pesquisa de natureza qualitativa. De acordo com Bicudo (2011), a abordagem fenomenológica permite a possibilidade de explorar, compreender e interpretar as características do fenômeno investigado a partir da interrogação formulada. Assim, nos debruçamos à exploração, compreensão e interpretação das características do que se entende por modelagem matemática nas publicações dos livros do ICTMA mediante uma pesquisa de natureza qualitativa por meio de uma abordagem fenomenológica.

A fenomenologia, interroga o fenômeno com vistas a ir-às-coisas-mesmas e percebê-las do modo como se manifestam, isto é, livre de preconcepções que estabeleçam o que é para ser visto. Ao inquirir o que se entende por modelagem matemática no contexto dos trabalhos publicados nos livros dos ICTMA procuramos olhar a coisa-mesma buscando modos de manifestação e explicitação dos autores dos trabalhos.

Assumir uma atitude fenomenológica significa assumir um papel de observador da cena de modo imparcial visto que "as coisas aparecem como elas são, e elas são como elas aparecem" (SOKOLOWSKI, 2014, p. 23). Assumir este papel significa fazer o que Bicudo (2011) denomina de redução fenomenológica e que, segundo essa autora, consiste numa suspensão ou ainda neutralização de nossas crenças e juízos atribuídos às coisas ao descrevê-las para que estas possam mostrar-se por si mesmas.

Para efetuar a descrição do material utilizado em nossa pesquisa, artigos dos livros ICTMA, foram necessárias diversas leituras de modo a buscar neles as coisas como elas se mostram, ou seja, o fenômeno é descrito sem agregar nossos entendimentos, interpretações ou referenciais teóricos prévios; ele é apenas relatado como se mostra tendo como pano de fundo a interrogação que move a investigação.

A busca pela estrutura do fenômeno se dá ao transcender o que está exposto nos artigos dos livros ICTMA e nas descrições que realizamos, necessitando de dois movimentos sucessivos de análise: a análise ideográfica e a análise nomotética. Para Bicudo (2011, p. 58) a análise ideográfica consiste no "emprego de ideogramas, ou seja, de expressões e ideias por meio de símbolos". É neste movimento de análise que ocorre o destaque de unidades de significado, que segundo Garnica (1997) podem ser entendidas como:

[...] recortes considerados significativos pelo pesquisador, dentre os vários pontos aos quais a descrição pode leva-lo. Para que as unidades significativas possam ser recortadas, o pesquisador lê os depoimentos à luz da sua interrogação por meio da qual pretende ver o fenômeno que é olhado de uma dentre as várias perspectivas possíveis (GARNICA,1997, p. 120). 
A partir do destaque das unidades de significado entra em cena a análise nomotética, cujo movimento analítico permite transcender as individualidades postas nas unidades de significado e buscar as convergências que estabelecem os denominados núcleos de ideias, desvelando a estrutura do fenômeno investigado (BICUD0, 2011).

No presente artigo, a nossa metodologia segue esse encaminhamento para análise de capítulos publicados nos livros dos ICTMA visando identificar o que entendem por modelagem matemática os autores dos capítulos analisados.

0 ICTMA, sigla em inglês para Conferência Internacional sobre o Ensino de Modelagem Matemática e Aplicações, teve sua primeira edição no ano de 1983 quando educadores, pesquisadores e estudantes interessados em Modelagem Matemática na Educação Matemática, organizaram-se em um grupo e, no mesmo ano, organizaram a conferência realizada na universidade de Exeter na Inglaterra.

A conferência se tornou bienal e passou a ser sediada por diferentes países do mundo. Cada edição desta conferência resulta na produção de um livro ICTMA que contém alguns dos trabalhos que foram apresentados e discutidos no evento.

0 material da presente pesquisa é constituído pelos livros das últimas cinco edições ICTMA, incluindo assim do ICTMA 13 ao ICTMA 17, publicados no período de 2010 a 2017 (Quadro 1). Nossa abrangência para estas edições da conferência visa buscar entendimento para o interrogado na pesquisa na última década.

Quadro 1 - Edições das Conferências ICTMA selecionadas para a análise.

\begin{tabular}{|c|c|c|l|}
\hline Edição & $\begin{array}{c}\text { Ano da } \\
\text { conferência }\end{array}$ & $\begin{array}{c}\text { Ano de publicação } \\
\text { do livro }\end{array}$ & \multicolumn{1}{c|}{ Local } \\
\hline $\begin{array}{c}\text { ICTMA 13 } \\
\text { Reunião via satélite }\end{array}$ & 2007 & 2010 & $\begin{array}{l}\text { Indiana University, Estados Unidos da América } \\
\text { Kathmandu University, Nepal }\end{array}$ \\
\hline ICTMA 14 & 2009 & 2011 & University of Hamburg, Alemanha \\
\hline ICTMA 15 & 2011 & 2013 & Australian Catholic University (Melbourne), Austrália \\
\hline ICTMA 16 & 2013 & 2015 & Universidade Regional de Blumenau, Brasil \\
\hline ICTMA 17 & 2015 & 2017 & University of Nottingham, Inglaterra \\
\hline
\end{tabular}

0 ICTMA é uma conferência que inclui temáticas relativas à modelagem e também temáticas relativas a aplicações da matemática. Todavia, nosso interesse está nos textos que se referem à modelagem matemática e, por essa razão selecionamos os artigos para compor o nosso material de análise considerando alguns critérios. Primeiramente selecionamos os capítulos que incluem o termo modelagem matemática no título ou no resumo. Em segundo lugar, destes que versavam sobre modelagem matemática, selecionamos aqueles cujos autores publicaram em pelo menos três edições diferentes do ICTMA no período de 2010-2017, visando buscar o que entendem por modelagem matemática pesquisadores ou professores que têm realizado pesquisa com modelagem com certa regularidade no período a que nos referimos. Por fim, destes que restaram dos dois critérios anteriores, selecionamos apenas os capítulos que incluem a descrição de uma atividade de modelagem matemática. Usando estes critérios selecionamos cinquenta e sete capítulos para compor a base para o detalhamento da investigação fenomenológica.

3 No ano de 2007 dois encontros foram realizados, porém apenas um livro - ICTMA 13, foi organizado. 
A partir da leitura dos capítulos selecionados, em cada capítulo foi identificada uma unidade de significado (US) relativa ao entendimento de modelagem matemática revelado no texto. Para codificar estas US usamos US.y.z em que y refere-se ao número do capítulo do ICTMA e z indica a edição do ICTMA a qual pertence o capítulo analisado. Assim, por exemplo, US.11.13 refere-se à unidade de significado relativa ao entendimento de modelagem matemática que está no capítulo onze no ICTMA 13.

A seguir apresentamos o manifestado nos capítulos analisados, apresentando unidades de significado ${ }^{4}$ para cada um dos núcleos de ideias resultantes da análise empreendida.

\section{OS ENTENDIMENTOS DE MODELAGEM MATEMÁTICA REVELADOS}

Inquirir o que se entende por modelagem matemática no contexto dos trabalhos publicados nos livros do ICTMA permitiu, por meio da análise ideográfica, o destaque de cinquenta e sete unidades de significado. Destas unidades, mediante a análise nomotética, constituímos quatro núcleos de ideias que expressam o que se entende por modelagem matemática a partir da análise dos livros dos ICTMA relativos ao período de 2007 a 2017.

Os núcleos de ideias resultantes dessa análise nomotética são: (1) Modelagem Matemática como forma de resolver um problema; (2) Modelagem Matemática como prática pedagógica; (3) Modelagem Matemática como possibilidade para resolver na sala de aula problemas autênticos; (4) Modelagem Matemática como possibilidade de promover o pensamento crítico. Considerando a quantidade de unidades de significado, selecionamos para incluir no artigo quatro delas para cada um dos quatro núcleos de ideias.

0 primeiro núcleo de ideias Modelagem Matemática como forma de resolver um problema reflete a convergência de vinte e três unidades de significado identificadas em vinte e três capítulos. É possível afirmar que o problema a que se referem os autores pode ser um problema da realidade, um problema que surgiu em uma disciplina escolar, mas não no âmbito da disciplina de matemática, ou mesmo os já reconhecidos problemas de palavra. Um recorte das unidades de significado relativas ao núcleo ideias Modelagem Matemática como forma de resolver um problema é apresentado no Quadro 2.

Quadro 2 - Unidades de significado do núcleo de ideias Modelagem Matemática como forma de resolver um problema.

\begin{tabular}{|c|l|}
\hline Código US & \multicolumn{1}{c|}{ Unidade de Significado } \\
\hline US.11.13 & $\begin{array}{l}\text { Na abordagem adotada neste texto a modelagem matemática é uma possiblidade para a resolução de problemas que sur- } \\
\text { gem nas áreas de outras disciplinas ou no mundo fora da escola. O problema pode ser sugerido pelos professores na sala } \\
\text { de aula. A modelagem matemática inicia e termina no problema do mundo real. }\end{array}$ \\
\hline US.52.13 & $\begin{array}{l}\text { A modelagem matemática inclui a resolução de um problema (iterativa ou cíclica) conforme ilustra a Figura } 52.1 \text { [0 autor } \\
\text { referia-se ao ciclo de modelagem em Borromeo Ferri, 2006, p. 92)]. }\end{array}$ \\
\hline US.28.16 & A realidade subjacente à modelagem matemática refere-se à resolução de problemas do mundo real. \\
\hline US.32.14 & $\begin{array}{l}\text { As ferramentas tecnológicas costumam ser úteis ou, às vezes, até necessárias para a modelagem matemática de uma } \\
\text { situação da realidade. Alguns problemas são resolvidos mais rapidamente ou até simplificados demasiadamente; outros só } \\
\text { podem ser resolvidos com o uso da tecnologia. Em conexão com isso, é importante discutir a influência de ferramentas da } \\
\text { tecnologia digital para resolver um problema de modelagem matemática }\end{array}$ \\
\hline
\end{tabular}

Fonte: dados da pesquisa

4 Considerando a quantidade de US e a possibilidade da extensão do artigo, apresentamos exemplares de US para cada núcleo de ideias. 
Ao entender a Modelagem Matemática como forma de resolver um problema, os capítulos retratam que a modelagem matemática elucida relações entre a realidade e a matemática, por meio da matematização, construção de um modelo matemático e interpretação e validação de resultados. Estes procedimentos são considerados em diversas caracterizações veiculadas na literatura da área. Alguns pesquisadores destacam o uso dos chamados ciclos de modelagem matemática como instrumentos para guiar os alunos no desenvolvimento das atividades de modelagem matemática (por exemplo DOERR; ÄRLEBÄCK; MISFELDT, 2017, entre outros). Estes ciclos referem-se a esquemas que visam caracterizar os diferentes procedimentos que seus autores identificam no desenvolvimento de uma atividade de modelagem matemática.

Em termos gerais, a atividade que inicia com uma situação-problema e viabiliza a obtenção de uma solução para um problema identificado nessa situação por meio de modelos matemáticos é chamada modelagem matemática nos entendimentos veiculados nos capítulos analisados. Este entendimento de modelagem matemática associado à resolução de problemas advindos de contextos reais também é veiculado na literatura nacional, por exemplo, na voz de Bassanezi (2002), um dos precursores da modelagem matemática no Brasil, que considera que a modelagem matemática é a arte de resolver problemas reais.

Este entendimento é, sobretudo, partilhado por diferentes autores e está, segundo Almeida e Vertuan (2011), na gênese da atividade de modelagem matemática. Este entendimento se revela também nos chamados ciclos de modelagem matemática que visam elucidar os diferentes procedimentos requeridos em uma atividade de modelagem matemática. Este aspecto de caracterizar o que se deve fazer em atividades de modelagem por meio de um ciclo de modelagem matemática também foi identificado na análise dos artigos, como ilustra, por exemplo, a US da segunda linha do Quadro 4.

0 que este núcleo de ideias também inclui é que ao compreender a modelagem matemática como forma de resolver um problema, as relações com a tecnologia digital são relevantes. A inserção das tecnologias digitais na modelagem matemática, não tem apenas o papel de facilitar a obtenção de modelos para a matematização da situação, mas seu uso tem relevância nas diversas etapas de resolução de uma atividade de modelagem, conforme sugerem Greefrath e Vorhölter (2016).

A convergência das unidades de significado que compõe cada núcleo foi feita tendo por norte a leitura dos trabalhos publicados nos livros do ICTMA no período de 2010 a 2017, levando em consideração as perspectivas adotadas pelos autores dos trabalhos. Neste contexto os artigos pertencentes a este primeiro núcleo visam discutir, argumentar, teorizar, de modo predominante sobre modelagem como forma de resolver um problema. Isto significa que, as unidades de significado, ao convergirem para 0 núcleo de ideias modelagem matemática como forma de resolver um problema, detalham 0 processo de modelagem com a predominância dos argumentos propostos por Blum (2002); apresentam questões investigativas associadas às transições de uma fase para outra nos ciclos de modelagem matemática (GALBRAITH et. al, 2007); elencam princípios para identificar como uma situação pode ser/ter potencial para o desenvolvimento de um modelo matemático (GALBRAITH, 2007). Assim, este núcleo de ideias tem como centro a modelagem matemática com o foco na resolução de um problema e no que isso implica para a atividade de modelagem matemática, ou seja, o foco está no procedimento e nas fases que permeiam a resolução de um problema de modelagem matemática.

0 segundo núcleo de ideias se constituiu por meio da convergência de vinte e duas unidades de significado identificadas e revela um entendimento de Modelagem Matemática como uma prática pedagógica. Neste núcleo inserem-se ideias relativas a significados do que é modelagem matemática em contextos de ensino e de aprendizagem, bem como o papel da metacognição e da comunicação 
no desenvolvimento de atividades de modelagem matemática na sala de aula. A ideia central é reconhecer e discutir o papel da modelagem matemática para ensinar e aprender matemática bem como estruturar ideias de como sujeitos aprendem a fazer modelagem matemática considerando a sua inserção em cursos de formação de professores, por exemplo. A perspectiva cognitiva da modelagem matemática, caracterizada em Kaiser e Sriraman, (2006) como aquela em que a finalidade da modelagem é discutir como os sujeitos aprendem ao fazer modelagem matemática, é referida em algumas unidades de significado incluídas neste núcleo de ideias. 0 Quadro 3 apresenta algumas das unidades de significado deste núcleo de ideias.

Quadro 3 - Unidades de significado do núcleo de ideias modelagem matemática como prática pedagógica.

\begin{tabular}{|c|l|}
\hline Código US & \multicolumn{1}{c|}{ Unidade de Significado } \\
\hline US.18.14 & $\begin{array}{l}\text { Na modelagem matemática a reflexão deve ser relacionada ao conteúdo matemático e às decisões por meio das quais o } \\
\text { conteúdo é evocado e implementado para resolver o problema que emerge da modelagem da situação. } 0 \text { interesse na refle- } \\
\text { xão refere-se ao seu papel em oportunizar a atividade metacognitiva no processo de modelagem. [...] 0 objetivo educacio- } \\
\text { nal é que os estudantes aprendam a resolver problemas e aprendam também a matemática usada nesta resolução. }\end{array}$ \\
\hline US.24.16 & $\begin{array}{l}\text { As sequências de desenvolvimento de modelos estão alocadas na perspectiva contextual da modelagem matemática } \\
\text { (Kaiser e Sriraman 2006), na qual os alunos são confrontados com atividades em que precisam aprender matemática para } \\
\text { compreender diferentes situações da realidade. }\end{array}$ \\
\hline US.22.15 & $\begin{array}{l}\text { De uma forma ampla, dois podem ser os objetivos pedagógicos da modelagem. O primeiro é a modelagem em si, sendo } \\
\text { o objetivo aprender a modelagem matemática de uma situação. [...] O segundo objetivo é a construção do conhecimento } \\
\text { matemático, sendo a modelagem tratada como um método para alcançar essa meta. }\end{array}$ \\
\hline US.20.14 & $\begin{array}{l}\text { As tarefas de modelagem escolar por natureza envolvem "vínculo(s) genuíno(s)" com algum contexto do mundo real que } \\
\text { requer algum nível de complexidade do pensamento matemático ao tentar resolver tais tarefas. }\end{array}$ \\
\hline
\end{tabular}

Fonte: dados da pesquisa

0 entendimento de modelagem matemática como prática pedagógica compreende que esta pode ser uma atividade que gera demandas cognitivas diversas. As discussões neste núcleo se voltam para a finalidade do uso da modelagem matemática na sala de aula. Este núcleo foi nomeado modelagem matemática como prática pedagógica, pois reúne unidades de significado dos textos cuja ideia predominante está associada à modelagem matemática voltada ao planejamento e às dinâmicas das aulas de matemática e outras componentes curriculares. As unidades de significado deste núcleo advém de textos que abordam novas maneiras de usar a modelagem matemática em sala de aula como, por exemplo, o ensino por meio da dual modelling (KAWAKAMI; SAEKI; MATSUZAKI, 2015); a aplicação de técnicas e ferramentas associadas à etnomatemática, como as propostas por Orey e Rosa (2015); e 0 uso de teorias, por exemplo, o papel da metacognição (FLAVELL, 1979) para a modelagem matemática no favorecimento da aprendizagem e das práticas pedagógicas com modelagem matemática.

Neste contexto, pelo menos dois encaminhamentos se evidenciaram nos textos. Um deles refere-se às argumentações de Galbraith (2012). Este autor faz uma distinção entre modelagem como conteúdo e modelagem como veículo. Quando utilizada como conteúdo, a modelagem matemática se propõe a capacitar os alunos a usar seu conhecimento matemático para resolver problemas reais e para continuar a desenvolver esta capacidade ao longo do tempo. Quando usada como um veículo, a principal finalidade das atividades de modelagem é possibilitar a aprendizagem de conceitos matemáticos ou processos que fazem parte da matemática curricular incluída nos programas (GALBRAITH, 2012). 
Outro encaminhamento refere-se às discussões com relação às diferentes perspectivas para 0 uso da modelagem matemática na sala de aula. Neste sentido, Blum (2015) ampliou 0 quadro de perspectivas de modelagem matemática apresentado em Kaiser e Sriraman (2006) e defende que podem se caracterizar perspectivas para a modelagem matemática por meio de um par (objetivo, exemplo adequado) que associa 0 objetivo do uso da modelagem matemática com as atividades escolhidas para a sala de aula em cada situação conforme ilustra 0 Quadro 4.

Quadro 4 - Perspectivas de Modelagem Matemática.

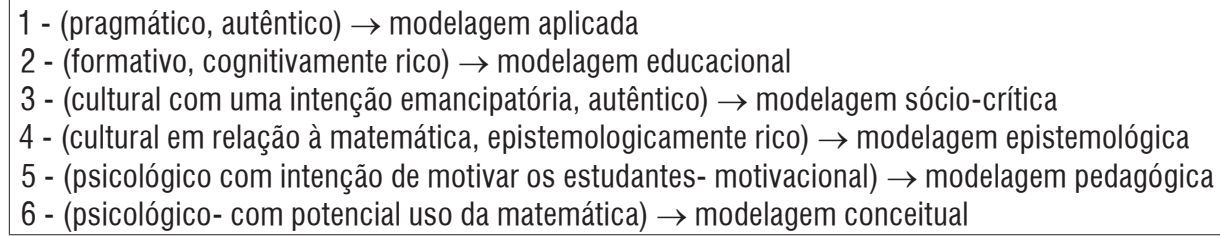

Fonte: Blum (2015, p. 82)

Assim, à luz da classificação das atividades proposta por Galbraith (2012) e as perspectivas caracterizadas por Blum (2015), a modelagem matemática como prática pedagógica envolve, além de ações didáticas e pedagógicas, a complexidade do sistema escolar e as diferenças entre a modelagem matemática profissional e a modelagem matemática escolar de modo a estreitar tal dicotomia.

Ao levarmos em conta que as atividades de modelagem matemática partem de situações reais identificamos, mediante a análise dos capítulos, a preocupação de alguns autores com a originalidade, veracidade e fidelidade das situações reais tratadas nas atividades de modelagem matemática na sala de aula, o que é diferente, por exemplo, da abordagem de problemas de palavras como problemas de modelagem matemática. Sete unidades de significado identificadas em $\underline{7}$ sete capítulos sinalizam ideias convergentes com a genuinidade das situações reais presentes nas atividades e constituem 0 terceiro núcleo de ideias: Modelagem Matemática como possibilidade para resolver na sala de aula problemas autênticos. 0 recorte de unidades de significados para este núcleo de ideias está alocado no Quadro 5.

Quadro 5 - Unidades de significado do núcleo de ideias Modelagem Matemática como possibilidade de resolver na sala de aula problemas autênticos.

\begin{tabular}{|c|l|}
\hline Código US & \multicolumn{1}{c|}{ Unidade de Significado } \\
\hline US.57.14 & $\begin{array}{l}\text { Com base em nossa extensa pesquisa empírica vemos a necessidade de tratar problemas autênticos de modelagem e que } \\
\text { promovam uma gama de competências de modelagem e ampliem o raio de ação dos estudantes. Esta abordagem tem } \\
\text { como ponto de partida que, para promover competências de modelagem, os alunos precisam ter suas próprias experiên- } \\
\text { cias com problemas autênticos de modelagem. }\end{array}$ \\
\hline US.46.15 & $\begin{array}{l}\text { A atividade de modelagem matemática relacionada à entrega de uma embalagem em um domicílio no Japão foi realizada } \\
\text { com vistas à resolução de um problema em um cenário real. [...] o propósito é introduzir tarefas autênticas de modelagem } \\
\text { matemática no ensino secundário. }\end{array}$ \\
\hline US.8.16 & $\begin{array}{l}\text { Quando uma atividade de modelagem contém aspectos autênticos, isso faz com que a atividade seja complexa, mas tam- } \\
\text { bém aberta e com demandas mais abrangentes dos alunos. }\end{array}$ \\
\hline US.20.17 & $\begin{array}{l}\text { A modelagem matemática autêntica inclui, entre outras coisas, que mesmo nas escolas, os próprios modeladores podem } \\
\text { encontrar a situação-problema e formular o(s) problema(s). Inspirados pelos resultados positivos em relação a problemas } \\
\text { de modelagem autênticos, começamos a pensar sobre uma atividade em que se torna necessário a reformulação de um } \\
\text { modelo matemático em uma situação do mundo real. }\end{array}$ \\
\hline
\end{tabular}

Fonte: dados da pesquisa 
0 núcleo de ideias relativo à Modelagem Matemática como possibilidade de resolver na sala de aula problemas autênticos pode ser descrito sob dois pontos de vista: um relacionado à perspectiva da modelagem aplicada em que a autenticidade da modelagem matemática refere-se aos problemas aplicados na indústria ou na ciência; 0 segundo aponta a autenticidade como associada a uma atividade de modelagem matemática em que a situação-problema advém de um contexto de fora da escola, ou seja, de contextos da realidade ou da cotidianidade da vida dos alunos. A discussão emergente está associada aos diferentes tipos de modelos matemáticos que emergem nas atividades, considerando sua relação com modelos utilizados por especialistas da área de aplicação da situação-problema. A autenticidade em atividades de modelagem matemática é oriunda da gểnese dos problemas, quando estes são advindos da experiência profissional, bem como do campo de trabalho dos profissionais.

A possibilidade de abordar problemas autênticos em sala de aula é vista por Lesh e Lamon (1992) como uma atividade de modelagem matemática que

[...] exige que os alunos usem uma amostra representativa dos conhecimentos e habilidades que refletem os níveis de competência direcionados no estudo e, ao mesmo tempo, as atividades devem evitar concepções estreitas, tendenciosas, obsoletas ou criadas em contra produtividade sobre a natureza das matemáticas, a natureza das situações realistas de resolução de problemas em que a matemática é útil e as variedades de capacidades matemáticas que são produtivas nessas situações (LESH; LAMON, 1992, p. 26, tradução nossa).

A modelagem matemática, sendo uma possibilidade de abordar problemas autênticos, está associada à capacidade de identificar questões, variáveis, relações ou suposições relevantes em uma situação do mundo real, bem como traduzi-las em matemática, interpretar e analisar a solução para a situação dada, analisando e comparando modelos, o que corrobora com a argumentação relativa ao desenvolvimento de competências em atividades de modelagem matemática conforme discute Maaß, (2006). Este entendimento pode indicar a viabilidade da proposição e formulação de situações-problema, o que vai ao encontro do argumento de Pollak (2012) ao mencionar que o coração da modelagem matemática está na formulação de problemas e a experiência com a matemática que sua resolução viabiliza aos estudantes.

Na literatura as discussões a respeito da definição de autenticidade e estudos acerca de quão autênticas são as atividades de modelagem matemática são veiculadas por diferentes pesquisadores (VOS, 2011). Alinhadas à concepção de autenticidade por meio de aspectos que aproximam a prática dos alunos da prática dos especialistas estão as ideias de Niss (1992) e mais tarde de Vos (2011). Para Niss (1992) em uma autêntica situação extramatemática é possível encontrar uma prática existente fora da matemática que trata de fenômenos ou problemas que são próprios dessa área e atestada por especialistas. Já para Vos (2011, p. 718) a autenticidade é um construto social mais amplo, que engloba também origens matemáticas para situações autênticas sob 0 argumento de que a certificação da situação autêntica pode ser oferecida por outros especialistas conhecedores do problema em questão.

É importante destacar que 0 entendimento de modelagem matemática veiculado traz consigo uma ampla gama de discussões a respeito da inclusão de situações autênticas no ambiente da sala de aula. Vos (2015), por exemplo, afirma que se as situações exploradas com os alunos fossem totalmente autênticas, os professores estariam trabalhando com a formação de cientistas e erros 
deveriam ser considerados graves, visto que um erro na solução de algum problema poderia causar graves consequências.

A abordagem sobre autenticidade em modelagem matemática bem como sobre a consideração de aspectos autênticos está associada aos textos que têm por norte, entre outros, os trabalhos de Pollak (1969) e Niss (1992). Nestes trabalhos a discussão predominante diz respeito à importância de manter as características da modelagem matemática herdadas da matemática aplicada, tendo, por exemplo, como ponto de partida para a atividade de modelagem matemática a experiência com problemas que se aproximam da realidade e apresentam poucas simplificações, com maior proximidade dos problemas tratados por modeladores profissionais. Assim, este núcleo reúne trabalhos, e por sua vez, unidades de significado, cujo entendimento adotado questiona o contexto das situações-problema, o rigor daquilo que se considera como uma situação-problema a ser modelada, trazendo à tona, por exemplo, críticas ao uso de problemas de palavras como problemas de modelagem, como as detalhadas por Galbraith (2012). Para promover a discussão sobre autenticidade, critérios são formulados, problemas autênticos são apresentados, e diferentes perspectivas sobre o que caracteriza autenticidade são apresentadas, por exemplo, as perspectivas sobre autenticidade defendidas por Palm (2007) e Vos (2011).

Para além do desenvolvimento de competências relacionadas à matemática e de modelagem matemática, os capítulos do ICTMA também indicam um entendimento da Modelagem Matemática como uma possibilidade de promover pensamento crítico. Este núcleo de ideias foi constituído por meio do destaque de cinco unidades de significado identificadas em cinco capítulos que dizem a respeito da modelagem matemática como meio para desenvolver habilidades críticas e reflexivas para compreensão de fenômenos físicos e sociais (Quadro 6).

Quadro 6 - Unidades de significado do núcleo de ideias modelagem matemática como possibilidade de promover pensamento crítico.

\begin{tabular}{|c|l|}
\hline Código US & \multicolumn{1}{c|}{ Unidade de Significado } \\
\hline US.30.15 & $\begin{array}{l}\text { Uma abordagem para o ensino usando modelagem matemática é feita por meio da adoção de uma perspectiva sócio-crítica } \\
\text { em que o objetivo é o desenvolvimento de uma compreensão crítica do mundo por meio da modelagem de fenômenos } \\
\text { físicos e sociais. }\end{array}$ \\
\hline US.32.16 & $\begin{array}{l}\text { A modelagem matemática é usada para analisar, simplificar e descrever os fenômenos do cotidiano das pessoas, a fim de } \\
\text { prever resultados ou modificar os fenômenos. Neste processo, o propósito da modelagem matemática torna-se a capacida- } \\
\text { de de desenvolver habilidades críticas que permitem aos professores e alunos analisar e interpretar dados, formular e testar } \\
\text { hipóteses e desenvolver e verificar a eficácia de modelos matemáticos. }\end{array}$ \\
\hline US.49.16 & $\begin{array}{l}\text { A modelagem matemática é destinada a ajudar os alunos a entender melhor o mundo e contribuir para a formação de uma } \\
\text { imagem adequada da matemática. }\end{array}$ \\
\hline US.48.17 & $\begin{array}{l}\text { A modelagem matemática tornou-se uma metodologia de ensino que possibilita o desenvolvimento de uma capacidade } \\
\text { crítica e reflexiva que pode engajar os alunos em um processo de ensino e aprendizagem contextualizado que Ihes permite } \\
\text { envolver-se na construção de soluções de problemas de relevância social. }\end{array}$ \\
\hline
\end{tabular}

Fonte: dados da pesquisa

Alguns capítulos evidenciam que a modelagem matemática tem como propósito fornecer aos alunos elementos para entender o papel da matemática na sociedade. Tal entendimento foi manifestado no núcleo de ideias Modelagem matemática como uma possibilidade de promover pensamento crítico, de modo que as unidades de significado elucidam a emergência da função crítica e social 
que a modelagem matemática pode exercer na formação dos alunos. A perspectiva de modelagem indicada caracterizada por Barbosa (2001) e disseminada por Kaiser e Sriraman (2006) e denominada sócio-crítica, pode ser associada a este núcleo de ideias. Este entendimento de modelagem matemática evoca o trabalho dos alunos com um problema ou situação escolhida por eles no qual se engajam em discussões críticas e reflexivas.

De modo geral, este núcleo se distingue por contemplar unidades de significado advindas de trabalhos que salientam que as questões sociais permitem que os alunos desenvolvam um posicionamento crítico em relação ao contexto do mundo que nos cerca, dos modelos e do processo de modelagem. Assim, a modelagem matemática é vista como uma possibilidade de estender a compreensão dos alunos especialmente de sua cultura e da vida social. Uma das perspectivas assinaladas por Kaiser e Sriraman (2006), a perspectiva sócio-crítica é evidenciada no entendimento de modelagem matemática e autores que teorizam acerca da perspectiva sócio-critica são usados para embasar a concepção de modelagem matemática apresentada, como Skovsmose (1990), Barbosa (2001), além de documentos curriculares internacionais que veiculam a matemática como intrinsicamente ligada ao aprimoramento da compreensão do mundo, bem como da qualidade da participação dos sujeitos na sociedade.

Em síntese, a pluralidade de ideias identificadas indica que os textos dos livros dos ICTMA revelam diferentes entendimentos para a modelagem matemática, considerando especificidades da situação-problema bem como a finalidade da sua introdução em aulas de matemática.

\section{CONSIDERAÇÕES FINAIS}

Neste artigo nos amparamos em uma perspectiva fenomenológica para investigar entendimentos de modelagem matemática. Assim, colocamos em suspensão nossos juízos, crenças e pressupostos de modo a explicitar este entendimento tal qual como este se mostra em capítulos de livros dos ICTMA. Conforme já argumentamos, nossa escolha por este evento se ampara na possiblidade de captar estes entendimentos a partir de uma diversidade de autores, considerando a internacionalidade e abrangência do evento.

A partir desta perspectiva de investigação manifestaram-se quatro núcleos de ideias que revelam que a modelagem matemática no contexto dos trabalhos publicados nos livros dos ICTMA pode ser entendida como forma de resolver um problema; como prática pedagógica; como possibilidade de resolver na sala de aula problemas autênticos; como possibilidade de promover pensamento crítico.

Ressaltamos que as unidades de significado foram constituídas tendo por norte 0 contexto, ou seja, cada um dos artigos que constituíram os dados coletados nas edições do ICTMA de 2010 a 2017. Assim, considerando este contexto, as unidades de significado visam detalhar especificidades do entendimento de modelagem matemática que predominam em cada artigo analisado. $\mathrm{E}$, ainda que, em sua individualidade as unidades possam aparentar não ser excludentes, a perspectiva na qual os autores dos trabalhos analisados se apoiam foi considerada para sua inserção no respectivo núcleo de ideias.

No trabalho de Klüber (2012) o autor captou o que denomina de diferentes compreensões sobre modelagem matemática em trabalhos nacionais. No presente artigo ampliamos estas discussões passando a considerar entendimentos de modelagem matemática em uma abrangência ampla como a proporcionada pelos livros dos ICTMA considerando a última década de realizações da conferência. $\mathrm{Na}$ Figura 1 ilustramos como estes entendimentos se constituíram, do ponto de vista cronológico, ao longo dos dez anos a que se refere nossa investigação. 
Figura 1 - Linha do tempo relativa à emergência e continuidade do que se entende por Modelagem Matemática nos anais do ICTMA

\begin{tabular}{|c|c|c|c|c|}
\hline ICTMA13 & ICTMA14 & ICTMA15 & ICTMA16 & ICTMA17 \\
\hline $\begin{array}{l}\text { Modelagem Matemática } \\
\text { como forma de resolver } \\
\text { um problema }\end{array}$ & $\begin{array}{l}\text { Modelagem Matemática } \\
\text { como forma de resolver } \\
\text { um problema }\end{array}$ & $\begin{array}{c}\text { Modelagem Matemática } \\
\text { como forma de resolver } \\
\text { um problema }\end{array}$ & $\begin{array}{c}\text { Modelagem Matemática } \\
\text { como forma de resolver } \\
\text { um problema }\end{array}$ & $\begin{array}{c}\text { Modelagem Matemática } \\
\text { como forma de resolver } \\
\text { um problema }\end{array}$ \\
\hline \multirow[t]{3}{*}{$\begin{array}{l}\text { Modelagem Matemática } \\
\text { como prática pedagógica }\end{array}$} & $\begin{array}{l}\text { Modelagem Matemática } \\
\text { como prática pedagógica }\end{array}$ & $\begin{array}{l}\text { Modelagem Matemática } \\
\text { como prática pedagógica }\end{array}$ & $\begin{array}{l}\text { Modelagem Matemática } \\
\text { como prática pedagógica }\end{array}$ & $\begin{array}{l}\text { Modelagem Matemática } \\
\text { como prática pedagógica }\end{array}$ \\
\hline & & $\begin{array}{l}\text { Modelagem Matemática } \\
\text { como possibilidade de } \\
\text { resolver na sala de aula } \\
\text { problemas autênticos }\end{array}$ & $\begin{array}{l}\text { Modelagem Matemática } \\
\text { como possibilidade de } \\
\text { resolver na sala de aula } \\
\text { problemas autênticos }\end{array}$ & $\begin{array}{l}\text { Modelagem Matemática } \\
\text { como possibilidade de } \\
\text { resolver na sala de aula } \\
\text { problemas autênticos }\end{array}$ \\
\hline & & $\begin{array}{c}\text { Modelagem Matemática } \\
\text { como possibilidade de } \\
\text { promover pensamento } \\
\text { crítico }\end{array}$ & $\begin{array}{l}\text { Modelagem Matemática } \\
\text { como possibilidade de } \\
\text { promover pensamento } \\
\text { crítico }\end{array}$ & $\begin{array}{c}\text { Modelagem Matemática } \\
\text { como possibilidade de } \\
\text { promover pensamento } \\
\text { crítico }\end{array}$ \\
\hline
\end{tabular}

Fonte: os autores

Podemos perceber que com a consolidação da área de Modelagem Matemática, entendimentos vieram se confirmando com o passar dos anos. A modelagem matemática como forma de resolver um problema e como prática pedagógica são entendimentos que perpassam todo o período analisado, visto que são entendimentos que tratam da gênese da modelagem matemática e do vínculo desta com ações pedagógicas e educativas, como a inserção de atividades de modelagem matemática no contexto escolar bem como a finalidade desta implementação.

A modelagem matemática como possibilidade de resolver na sala de aula problemas autênticos e como possibilidade de promover pensamento crítico se mostrou nas três últimas edições do evento a que nos referimos no artigo. Estes entendimentos decorrem da necessidade de se reconhecer a importância de práticas de modelagem matemática cujas atividades mantenham a originalidade, veracidade e fidelidade das situações reais e que possam contribuir com o desenvolvimento de uma compreensão crítica do mundo, reconhecendo a função crítica e social da modelagem matemática na formação dos alunos.

Estes núcleos de ideias esboçam faces do fenômeno investigado sob diferentes enfoques, que fundamentados em teorias ou em práticas de sala de aula revelam, por um lado, o que tem sido veiculado em relação à modelagem matemática na área de Educação Matemática. Por outro lado, entretanto, interrogando o que se entende por modelagem matemática no contexto dos trabalhos publicados nos livros dos ICTMA?, os núcleos de ideias resultantes de uma leitura indo-às-coisas nos levam a ponderar que não parece possível desvincular o que se entende por modelagem matemática com qual é a finalidade da introdução da modelagem em aulas de matemática. De fato, o olhar para os artigos a partir da busca pelo entendimento do que é modelagem matemática, nos traz mais indícios do objetivo de cada autor com a inclusão de atividades de modelagem nas aulas a que se referem os autores em cada uma das pesquisas descritas nos artigos que analisamos do que propriamente um entendimento relativo ao o que é modelagem matemática.

Assim, enquanto Kaiser e Sriraman (2006) caracterizam perspectivas de modelagem matemática para a sala de aula, Blum (2015) já associa essas perspectivas com características que os 
problemas que emergem das atividades de modelagem devem ter em cada uma destas perspectivas. A presente pesquisa nos permite confirmar a assertiva de Blum (2015) a partir da leitura de cinquenta e sete artigos publicados em livros dos ICTMA. Identificando o que se entende por modelagem matemática dentro dessa região de inquérito específica (anais dos ICTMA), colocamos em discussão com a literatura os achados (quatro núcleos de ideias) e podemos inferir que eles revelam finalidades da inclusão de atividades de modelagem matemática nas práticas docentes em aulas de matemática.

\section{REFERÊNCIAS}

ALMEIDA, L. M. W.; VERTUAN, R. E. Discussões sobre 'como fazer' Modelagem na sala de aula. In: ALMEIDA, L. M. W.; ARAÚJO, J. de L.; BISOGNIN, E. (Org.). Práticas de Modelagem Matemática na Educação Matemática. 1. ed. Londrina: EDUEL - Editora da Universidade Estadual de Londrina, 2011, p. 19-44.

ALMEIDA, L. M.W.; SILVA, K. P. The Meaning of the Problem in a Mathematical

Modelling Activity. In: STILLMAN, G. A.; BLUM, W.; BIEMBENGUT, M. S. (Eds.). Mathematical Modelling in Education Research and Practice: Cultural, Social and Cognitive Influences. Cham: Springer, 2015, p. 45-54.

ARAÚJO, J. L.; CAMPOS, I. S. Negotiating the Use of Mathematics in a Mathematical

Modelling Project. In: STILLMAN, G. A.; BLUM, W.; BIEMBENGUT, M. S. (Eds.). Mathematical Modelling in Education Research and Practice: Cultural, Social and Cognitive Influences. Cham: Springer, 2015, p. 283-292.

BARBOSA, J. C. The Students' Discussions in the Modeling Environment. In: LESH, R.; GALBRAITH, P.; HAINES, C.; HURFORD, A. (Eds) Modeling Students' Mathematical Modeling Competencies: ICTMA 13. Boston: Springer, 2010, p. 365-372.

BARBOSA, J. C. Modelagem matemática e os professores: a questão da formação. Bolema, Rio Claro, v. 14, n. 15, p. 5-23, 2001.

BASSANEZI, R. C. Ensino-Aprendizagem com Modelagem Matemática. Editora Contexto, São Paulo, p. 1-386, 2002.

BICUDO, M. A. V. Pesquisa qualitativa segundo a visão fenomenológica. São Paulo: Cortez, p. 5-150, 2011.

BICUDO, M. A. V.; KLUBER, T. E. Pesquisa em Modelagem Matemática no Brasil: a caminho de uma metacompreensão. Cadernos de Pesquisa, v.41 n.144, 2011, p. 904-927.

BLUM, W. ICMI Study 14: Applications And Modelling In Mathematics Education - Discussion Document. Educational Studies in Mathematics. v. 51, 2002, p. 149-171.

BLUM, W. Quality teaching of mathematical modelling: What do we know, what can we do? In: The Proceedings of the 12th International Congress on Mathematical Education: Intellectual and Attitudinal Changes. New York: Springer, 2015, p. 73-96.

BORBA, M. "Can Modelling Be Taught and Learnt?” - A Commentary. In: KAISER, G.; BLUM, W.; FERRI, R. B.; STILLMAN, G. (Eds) Trends in Teaching and Learning of Mathematical Modelling: ICTMA 14. Dordrecht: Springer, 2011, p. 31-36. 
D'AMBROSIO, U. Mathematical Modelling as a Strategy for Building-Up Systems of Knowledge in Different Cultural Environments. In: STILLMAN, G. A.; BLUM, W.; BIEMBENGUT, M. S. (Eds.). Mathematical Modelling in Education Research and Practice: Cultural, Social and Cognitive Influences. Cham: Springer, 2015, p. 35-44.

DOERR, H. M.; ÄRLEBÄCK, J. B.; MISFELDT, M. Representations of Modelling in Mathematics Education. In: STILLMAN, G.; BLUM, W.; KAISER, G. (Eds.). Mathematical Modelling and Applications: International Perspectives on the Teaching and Learning of Mathematical Modelling. Cham: Springer, 2017, p. 71-81.

FLAVELL, J. H. Metacognition and cognitive monitoring: A new area of cognitive-developmental inquiry. American Psychologist, v.34, n. 10, p. 90-911, 1979.

FERRI, R. B. Theoretical and empirical differentiations of phases in the modelling process. ZDM, v. 38, n. 2, p. 86-95, 2006.

GALBRAITH, P. Dreaming a 'possible dream': More windmills to conquer. In: C. HAINES, C.; GALBRAITH, P.; BLUM, W.; KHAN, S. (Eds.). Mathematical Modelling: Education, Engineering and Economics. Chichester, UK: Horwood, p. 44-62, 2007.

GALBRAITH, P.; STILLMAN, G.; BROWN, J.; EDWARDS, I. Facilitating Middle Secondary Modelling Competencies. In: HAINES, C.; GALBRAITH, P.; BLUM, W.; KHAN, S. Mathematical Modelling Education, Engineering and Economics-ICTMA 12, p. 130-140, 2007.

GALBRAITH, P. Models of Modelling: genres, purposes or perspectives. Journal of Mathematical Modelling and Applications, Blumenau, v. 1, n. 5, p. 3-16, 2012.

GARNICA, A. V. M. Algumas notas sobre pesquisa qualitativa e fenomenologia. Interface - Comunicação, Saúde e Educação, São Paulo, v. 1, n. 1, p. 109-122, 1997.

JABLONKA, E.; WAGNER, D.; WALSHAW, M. Theories for studying social, political and cultural dimensions of mathematics education. In: CLEMENTS, M. A.; BISHOP, A. J.; KEITEL, C.; KILPATRICK, J.; LEUNG, F. K. S. (Eds.). Third International Handbook of Mathematics Education. Springer Science+Business Media B.V. p. 41-67, 2013.

KAISER, G.; SRIRAMAN, B. A global survey of international perspectives on modelling in mathematics education. ZDM, v. 38, n. 3, p. 302-310, 2006.

KAWAKAMI, T.; SAEKI, A.; MATSUZAKI, A. Necessity For Modelling Teaching Corresponding To Diversities: Experimental Lessons Based On Dual Modelling Cycle Framework For The 5 Th Grade Pupils. In: STILLMAN, G. A.; BLUM, W.; BIEMBENGUT, M. S. (Eds.). Mathematical Modelling in Education Research and Practice: Cultural, Social and Cognitive Influences. Cham: Springer, 2015, p. 1-32.

KLÜBER, T. E. Uma metacompreensão da modelagem matemática na educação matemática. 2012. 396 f. Tese (doutorado) - Universidade Federal de Santa Catarina, Programa de Pós-Graduação em Educação Científica e Tecnológica. Florianópolis, SC, 2012.

LUNA, A. V. A.; SOUZA, E. G.; LIMA, L. B. S. Mathematical Texts in a Mathematical Modelling Learning Environment in Primary School. In: STILLMAN, G. A.; BLUM, W.; BIEMBENGUT, M. S. (Eds.). Mathematical Modelling in Education Research and Practice: Cultural, Social and Cognitive Influences. Cham: Springer, 2015, p. 535-544. 
MAA $\beta$, K. What are modelling competences? ZDM, v. 38, n. 2, p. 113-142, 2006.

NISS, M. Applications and modelling in school mathematics - Directions for future development. Roskilde: IMFUFA Roskilde Universitetscenter, 1992.

OLIVEIRA, A. M. P.; BARBOSA, J. C. Mathematical Modelling, Mathematical Content and Tensions in Discourses. In: STILLMAN, G.; KAISER, G.; BLUM, W.; BROWN, J. (Eds) Teaching Mathematical Modelling: Connecting to Research and Practice. Dordrecht: Springer, 2013, p. 67-76.

OREY, D. C.; ROSA, M. Modelling the Wall: The Mathematics of the Curves on the Wall of Colégio Arquidiocesano in Ouro Preto. In: STILLMAN, G. A.; BLUM, W.; BIEMBENGUT, M. S. (Eds.). Mathematical Modelling in Education Research and Practice: Cultural, Social and Cognitive Influences. Cham: Springer, 2015, p.593-604.

PALHARINI, B. N.; ALMEIDA, L. M.W. Mathematical Modelling Tasks and the Mathematical Thinking of Students. In: STILLMAN, G. A.; BLUM, W.; BIEMBENGUT, M. S. (Eds.). Mathematical Modelling in Education Research and Practice: Cultural, Social and Cognitive Influences. Cham: Springer, 2015, p. 219-228.

PALHARINI, B. N.; TORTOLA, E.; ALMEIDA, L. M. W. Mathematical Modelling and Proof by Recurrence: An Analysis from a Wittgensteinian Perspective. In: STILLMAN, G.; BLUM, W.; KAISER, G. (Eds.). Mathematical Modelling and Applications: International Perspectives on the Teaching and Learning of Mathematical Modelling. Cham: Springer, 2017, p. 129-140.

POLLAK, H. 0. What is mathematical modeling? In: Mathematical Modelin Handbook. Bedfort: COMAP, 2012.

PALM, T. Features and impact of the authenticity of applied mathematical school tasks. In: BLUM, W. et al. (Eds.). Modelling and applications in mathematics education. New York: Springer. 2007. p. 201-208.

ROSA, M.; OREY, D. C. Ethnomodelling as the Mathematization of Cultural Practices. In: STILLMAN, G., BLUM, W., KAISER, G. (Eds.). Mathematical Modelling and Applications: International Perspectives on the Teaching and Learning of Mathematical Modelling. Cham: Springer, 2017, p. 153- 162.

SILVEIRA, E. Modelagem Matemática em Educação no Brasil: entendendo o Universo de Teses e Dissertações. Curitiba, 2007, 197p. Dissertação (Mestrado em Educação) - Universidade Federal do Paraná, Curitiba, 2007.

SKOVSMOSE, 0. Reflective knowledge: its relation to the mathematical modellig process. Int. J. Math. Educ. Sci. Technol., London, v. 21, n. 5, p. 765-779, 1990.

SOKOLOWSKI, R. Introdução à Fenomenologia. Tradução Alfredo de Oliveira Moares. 4a Edição. São Paulo: Edições Loyola, 2014, p.7-247.

SOUZA, E. G.; ALMEIDA, L. M. W.; KLÜBER, T. E. Research on Mathematical Modelling in Mathematics Education in Brazil: Overview and Considerations. In: RIBEIR0, A. J.; HEALY, L.; BORBA, R. E. S.; FERNANDES, S. H. A. A. (Eds.). Mathematics Education in Brazil: Panorama of Current Research. Cham: Springer, 2018, p. 211-228.

SRIRAMAN, B.; NARDI, E. Theories in mathematics education: Some developments and ways forward. In: CLEMENTS, M. A. et al. (Eds.) Third international handbook of mathematics education. New York: Springer, 2013, p. 303-325. 
STILLMAN, G. A.; BLUM, W.; BIEMBENGUT, M. S. Cultural, Social, Cognitive and Research Influences on Mathematical Modelling Education. In: STILLMAN, G. A.; BLUM, W.; BIEMBENGUT, M. S. (Eds.). Mathematical Modelling in Education Research and Practice: Cultural, Social and Cognitive Influences. Cham: Springer, 2015, p. 1-32.

VOS, P. Authenticity in Extra-curricular Mathematics Activities: Researching Authenticity as a Social Construct. In: STILLMAN, G. A.; BLUM, W.; BIEMBENGUT, M. S. (Eds.). Mathematical Modelling in Education Research and Practice Cultural, Social and Cognitive Influences. Cham: Springer, 2015, p. 105-113.

VOS, P. What Is 'Authentic' in the Teaching and Learning of Mathematical Modelling? In: KAISER, G.; BLUM, W.; FERRI, B. R.; STILLMAN, G. (Eds.). Trends in Teaching and Learning of Mathematical Modelling: ICTMA 14. Dordrecht: Springer, 2011, p. 713-722.

RECEBIDO EM: 16 set. 2020

CONCLUÍDO EM: 11 jan. 2021 